\title{
Asymmetric exclusion model with impurities
}

\author{
Matheus J. Lazo ${ }^{1, *}$ and Anderson A. Ferreira ${ }^{2}$ \\ ${ }^{1}$ Instituto de Matemática, Estatística e Física-FURG, Rio Grande, RS, Brazil \\ ${ }^{2}$ Instituto de Física Teórica, UNESP, São Paulo, SP, Brazil
}

(Received 18 August 2009; revised manuscript received 5 May 2010; published 20 May 2010)

\begin{abstract}
An integrable asymmetric exclusion process with impurities is formulated. The model displays the full spectrum of the stochastic asymmetric XXZ chain plus new levels. We derive the Bethe equations and calculate the spectral gap for the totally asymmetric diffusion at half filling. While the standard asymmetric exclusion process without impurities belongs to the KPZ universality class with an exponent $\frac{3}{2}$, our model has a scaling exponent $\frac{5}{3}$.
\end{abstract}

DOI: 10.1103/PhysRevE.81.050104

PACS number(s): 02.50.Ey, 75.10.Pq, 02.30.Ik

One-dimensional three-state quantum Hamiltonians and master equations of reaction-diffusion processes have played an important role in describing strongly correlated electrons and nonequilibrium statistical mechanics in the last decades, mainly due to their intrinsic and nontrivial many-body behavior. Remarkably, in one dimension several models in this category are exactly solvable, as the spin-1 Sutherland [1] and $t-J$ [2] models, and the asymmetric diffusion of two types of particles [3]. In its formulation in terms of particles with two global conservation laws, these models describe the dynamics of two types of particles on the lattice, where the total number of particles of each type is conserved separately. In order to ensure integrability, all known models in this class satisfy some particle-particle exchange symmetries [4,5]. Recently, we introduced a new class of three-state model in the context of high-energy physics that is integrable despite it does not have particle-particle exchange symmetry [5]. The quantum version is closely related to the strong regime of the $t-U$ Hubbard model and the XXC model [6]. In this work we formulate a stochastic model related to [5] that describes an asymmetric exclusion process (ASEP) with impurities. Although our model can be solved by the coordinate Bethe ansatz, we are going to formulate a matrix product ansatz (MPA) [4,7] due its simplicity and unifying implementation for arbitrary systems. This MPA introduced in $[4,7]$ can be seen as a matrix product formulation of the coordinate Bethe ansatz and it is suited to describe all eigenstates of integrable models. We solve this model with periodic boundary condition through the MPA and we analyze the spectral gap for some special cases. Our model displays the full spectrum of the ASEP without impurities [8] plus new levels. The first excited state belongs to these new levels and has unusual scaling exponents. Although the ASEP without impurities belongs to the KPZ universality class [9] (dynamic exponent $\frac{3}{2}$ ), our model displays a scaling exponent $\frac{5}{2}$.

The model we propose describes the dynamics of two types of particles (types 1 and 2) on the lattice, where the total number of particles of each type is conserved. In this model if the neighbor sites are empty, particles of type 1 can jump to the right or to the left with rates $\Gamma_{0}^{1} 0_{1}^{0}$ and $\Gamma_{1}^{0} 1$, respectively. Particles of type 2 "impurities" do not jump to

\footnotetext{
*matheuslazo@furg.br
}

the neighbor sites if they are empty, but can change positions with neighbor particles of type 1 with rates $\Gamma_{2}^{1}{ }_{1}^{2}$ and $\Gamma_{1}^{2} \frac{1}{2}$ if particle 1 is on the left or on the right, respectively. There is no particle-particle exchange symmetry because particles 1 and 2 have different kinds of dynamics. The stochastic model we consider on a ring of perimeter $L$ is related to the Hamiltonian $H=H^{(0)}+H^{(2)}$, where $(\alpha=0,2)$

$$
\begin{aligned}
H^{(\alpha)}= & \sum_{j=1}^{L}\left[\Gamma_{\alpha}^{1}{ }_{1}^{\alpha}\left(E_{j}^{\alpha, 1} E_{j+1}^{1, \alpha}-E_{j}^{1,1} E_{j+1}^{\alpha, \alpha}\right)\right. \\
& +\Gamma_{1 \alpha}^{\alpha} \frac{\alpha}{1}\left(E_{j}^{1, \alpha} E_{j+1}^{\alpha, 1}-E_{j}^{\alpha, \alpha} E_{j+1}^{1,1}\right),
\end{aligned}
$$

and $E^{\alpha, \beta}(\alpha, \beta=0,1,2)$ are the usual $3 \times 3$ Weyl matrices with $i, j$ elements $\left(E^{l, m}\right)_{i, j}=\delta_{l, i} \delta_{m, j}$, and $\Gamma_{\alpha}^{1} \alpha_{1}^{\alpha}+\Gamma_{1 \alpha}^{\alpha}{ }_{\alpha}^{1}=1$. Finally, model (1) is related to the tracer diffusion model [4] by an interchange of labels 0 and 1; however, the exact solutions of these models describe different sectors of particle occupations.

The conservation of particles supplemented by the periodic boundary condition of Eq. (1) implies that the total numbers of particles $n_{1}, n_{2}$ on classes 1 and 2 as well the momentum $P=\frac{2 \pi l}{L} \quad(l=0,1, \ldots, L-1)$ are good quantum numbers. The eigenstates $|\Psi\rangle$ of the eigenvalue equation $H|\Psi\rangle=\varepsilon^{n_{1}, n_{2}}|\Psi\rangle$ belonging to the eigensector labeled by $\left(n_{1}, n_{2}, P\right)$ are given by

$$
|\Psi\rangle=\sum_{\{\alpha\},\{x\}} f\left(x_{1}, \alpha_{1} ; \ldots ; x_{n}, \alpha_{n}\right)\left|x_{1}, \alpha_{1} ; \ldots ; x_{n}, \alpha_{n}\right\rangle,
$$

where the kets $\left|x_{1}, \alpha_{1} ; \ldots ; s_{n}, \alpha_{n}\right\rangle$ denote the configurations with particles of type $\alpha_{i}\left(\alpha_{i}=1,2\right)$ located at the position $x_{i}$ $\left(x_{i}=1, \ldots, L\right)$ and $n=n_{1}+n_{2}$ is the total number of particles. The summation $\{\alpha\}=\left\{\alpha_{1}, \ldots, \alpha_{n}\right\}$ extends over all the permutations of $n$ numbers $\{1,2\}$ in which $n_{1}$ terms have the value of 1 and $n_{2}$ terms the value of 2 , while the summation $\{x\}$ $=\left\{x_{1}, \ldots, x_{n}\right\}$ extends, for each permutation $\{\alpha\}$, into the set of the nondecreasing integers satisfying $x_{i+1} \geq x_{i}+1$.

The MPA asserts that the amplitudes of an arbitrary eigenfunction (2) can be mapped to a matrix product

$$
\begin{aligned}
& f\left(x_{1}, \alpha_{1} ; \ldots ; x_{n}, \alpha_{n}\right) \\
& \quad \Leftrightarrow E^{x_{1}-1} A^{\left(\alpha_{1}\right)} E^{x_{2}-x_{1}-1} A^{\left(\alpha_{2}\right)} \cdots E^{x_{n}-x_{n-1}-1} A^{\left(\alpha_{n}\right)} E^{L-x_{n}},
\end{aligned}
$$

where for this map we can choose any operation on the matrix products that give a nonzero $c$ number [7]. The matrices 
$A^{(\alpha)}$ are associated with the particles of type $\alpha(\alpha=1,2)$ and the matrix $E$ is associated with the vacant sites [7]. Since the eigenfunction (2) has a well-defined momentum $P=\frac{2 \pi l}{L}$ due to the periodic boundary condition, the matrix product (3) should satisfy the constraints $f\left(x_{1}, \alpha_{1} ; \ldots ; x_{n}, \alpha_{n}\right)=e^{-i P} f\left(x_{1}\right.$ $\left.+1, \alpha_{1} ; \ldots ; x_{n}+1, \alpha_{n}\right)$ for $x_{n}<L$, and $f\left(x_{1}, \alpha_{1} ; \ldots ; x_{n}, \alpha_{n}\right)$ $=e^{-i P} f\left(1, \alpha_{n} ; \ldots ; x_{n-1}+1, \alpha_{n-1}\right)$ for $x_{n}=L$. Let us consider initially the simple cases where $n=1$ and $n=2$.

$n=1$. Since the Hamiltonian (1) is diagonal for $n_{1}=0$, let us consider only the case $n_{1}=1$. By inserting the ansatz (3) in Eq. (2), the eigenvalue equation gives us

$$
\begin{aligned}
& \varepsilon^{1,0} E^{x-1} A^{(1)} E^{L-x} \\
& =\Gamma_{0}^{1}{ }_{1}^{0} E^{x-2} A^{(1)} E^{L-x+1}+\Gamma_{1}^{0}{ }_{0}^{1} E^{x} A^{(1)} E^{L-x-1}-E^{x-1} A^{(1)} E^{L-x} .
\end{aligned}
$$

A convenient solution of Eq. (4) is obtained by introducing the spectral-parameter-dependent matrix $A^{(1)}=E A_{k}^{(1)}$ with $E A_{k}^{(1)}=e^{i k} A_{k}^{(1)} E$ and $k \in \mathrm{C}$. Inserting these relations into Eq. (4) we obtain

$$
\varepsilon(k) \equiv \varepsilon^{1,0}=\Gamma_{0}^{1}{ }_{1}^{0} e^{-i k}+\Gamma_{1}^{0}{ }_{0}^{1} e^{i k}-1 .
$$

The up to now free spectral parameter $k$ is fixed by imposing the boundary condition. This will be done only for the general $n$.

$n=2$. For two particles of types $\alpha_{1}$ and $\alpha_{2}\left(\alpha_{1}, \alpha_{2}=1,2\right)$ we have two kinds of relations coming from the eigenvalue equation. The configurations where the particles are at positions $(x, y)$ with $y-x=d>1$ give us the generalization of Eq. (4), and the configurations where the particles are at the colliding positions $(y=x+1)$ give us

$$
\begin{aligned}
& \varepsilon^{\alpha_{1}, \alpha_{2}} E A^{\left(\alpha_{1}\right)} A^{\left(\alpha_{2}\right)} E
\end{aligned}
$$

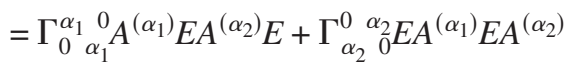

$$
\begin{aligned}
& +\Gamma_{\alpha_{1}}^{\alpha_{2} \alpha_{2}} \alpha_{1} E A^{\left(\alpha_{2}\right)} A^{\left(\alpha_{1}\right)} E-\left(\Gamma_{\alpha_{1} \alpha_{1}}^{0 \alpha_{1}}+\Gamma_{0 \alpha_{2}}^{\alpha_{2}{ }^{0}}+\Gamma_{\alpha_{2}}^{\alpha_{1} \alpha_{1}} \alpha_{2}\right) \\
& \times E A^{\left(\alpha_{1}\right)} A^{\left(\alpha_{2}\right)} E \text {, }
\end{aligned}
$$

where we introduced $\Gamma_{1}^{1} \frac{1}{1}=\Gamma_{2}^{2}{ }_{2}^{2}=\Gamma_{0}^{2}{ }_{2}^{0}=\Gamma_{2}^{0} \quad{ }_{0}^{2}=0$ and $\varepsilon^{0,2}=0$. A solution of these relations is obtained by identifying $A^{(\alpha)}$ as composed of $n_{\alpha}$ spectral-parameter-dependent matrices $A_{k_{1}^{(\alpha)}}^{(\alpha)}$ and $A_{k_{2}^{(\alpha)}}^{(\alpha)}[10]$, i.e.,

$$
A^{(\alpha)}=\sum_{j=1}^{n_{\alpha}} E A_{k_{j}^{(\alpha)}}^{(\alpha)}, \quad \text { with } E A_{k_{j}^{(\alpha)}}^{(\alpha)}=e^{i k_{j}^{(\alpha)}} A_{k_{j}^{(\alpha)}}^{(\alpha)} E,
$$

with $\alpha=1,2$ and $j=1, \ldots, n_{\alpha}$. By inserting Eq. (7) in the first kind of relations, where $y-x=d>1$, we obtain the energy in terms of the spectral parameters,

$$
\varepsilon^{n_{1}, n_{2}}=\sum_{j=1}^{n_{1}} \varepsilon\left(k_{j}^{(1)}\right),
$$

where $\varepsilon(k)$ is given by Eq. (5). Let us consider now Eq. (6) in the case where the particles are of the same type. Using Eqs. (7) and (8) in Eq. (6) for $\alpha_{1}=\alpha_{2}=1$ we obtain the relations

$$
A_{k_{j}}^{\left(\alpha_{1}\right)} A_{k_{l}^{\left(\alpha_{2}\right)}}^{\left(\alpha_{2}\right)}=S_{\alpha_{1} \alpha_{2}}^{\alpha_{1} \alpha_{2}}\left(k_{j}^{\left(\alpha_{1}\right)}, k_{l}^{\left(\alpha_{2}\right)}\right) A_{k_{l}^{\left(\alpha_{2}\right)}}^{\left(\alpha_{2}\right)} A_{k_{j}^{\left(\alpha_{1}\right)}}^{\left(\alpha_{1}\right)},
$$

where $\left(A_{k_{j}}^{\left(\alpha_{j}\right)}\right)^{2}=0$ and

$$
S_{1}^{1}{ }_{1}^{1}\left(k_{j}^{(1)}, k_{l}^{(1)}\right)=-\frac{\Gamma_{0}^{1} \frac{0}{1}+\Gamma_{1}^{0}{ }_{0}^{1} e^{i\left(k_{j}^{(1)}+k_{l}^{(1)}\right)}-e^{i k_{j}^{(1)}}}{\Gamma_{0}^{1}{ }_{1}^{0}+\Gamma_{1}^{0}{ }_{0}^{1} e^{i\left(k_{j}^{(1)}+k_{l}^{(1)}\right)}-e^{i k_{l}^{(1)}}} .
$$

For two impurities at "colliding" positions, the eigenvalue equation does not fix a commutation relation between the matrices $A_{k_{j}^{(2)}}^{(2)}$. On the other hand, the sum

$$
\sum_{j, l=1}^{2} A_{k_{j}^{(2)}}^{(2)} E^{d} A_{k_{l}^{(2)}}^{(2)} \neq 0,
$$

where the number of vacant sites between the impurities $d$ $=y-x$ is a conserved charge of the Hamiltonian (1), needs to be different from zero or the MPA will produce an eigenfunction with null norm. Moreover, the algebraic expression in Eq. (7) assures that any matrix product defining our ansatz (3) can be expressed in terms of two single matrix products $A_{k_{1}^{(2)}}^{(2)} A_{k_{2}^{(2)}}^{(2)} E^{L}$ and $A_{k_{2}^{(2)}}^{(2)} A_{k_{1}^{(2)}}^{(2)} E^{L}$. Using Eq. (7) we have, from the periodic boundary condition,

$$
A_{k_{j}^{(2)}}^{(2)} A_{k_{l}^{(2)}}^{(2)} E^{L}=e^{-i k_{j}^{(2)} L} e^{-i k_{l}^{(2)} L} A_{k_{j}^{(2)}}^{(2)} A_{k_{l}^{(2)}}^{(2)} E^{L} .
$$

To satisfy this equation we should have $k_{2}^{(2)}=-k_{1}^{(2)}+2 \pi j / L$ $(j=0,1, \ldots, L-1)$. Consequently, from Eq. (12) the most general commutation relation between the matrices $A_{k^{(2)}}^{(2)}$ and $A_{k_{2}^{(2)}}^{(2)}$ can be reduced to $A_{k_{1}^{(2)}}^{(2)} A_{k_{2}^{(2)}}^{(2)}=A_{k_{2}^{(2)}}^{(2)} A_{k_{1}^{(2)}}^{(2)}\left[\right.$ or $S_{2}^{2}{ }_{2}^{2}\left(k_{j}^{(2)}, k_{l}^{(2)}\right)$ $=1$ in Eq. (9)] by an appropriate change of variable in the spectral parameter $k_{1}^{(2)}$. Inserting these results in the sum of Eq. (11) and by imposing that the sum is not zero, we obtain $\left(j, l, v=1, \ldots, n_{2}\right)$

$$
k_{l}^{(2)} \neq k_{j}^{(2)}+\frac{\pi(2 m+1)}{d_{v}} \quad(m=0,1, \ldots),
$$

where $\left\{d_{v}\right\}$ is the set of all numbers of vacant sites between the impurities.

Let us consider now the case where the particles are of distinct species. By using Eqs. (5), (8), and (7), Eq. (6) gives us two independent relations for $\alpha_{1} \neq \alpha_{2}=1,2$ :

$$
\begin{aligned}
& {\left[\Gamma_{0}^{\alpha_{2} \alpha_{2}{ }^{0}}+\Gamma_{\alpha_{1} \alpha_{1}}^{0} e^{i\left(k^{\left(\alpha_{1}\right)}+k^{\left(\alpha_{2}\right)}\right)}-t_{\alpha_{1} \alpha_{2}} e^{i k^{\left(\alpha_{2}\right)}}\right] A_{k^{\left(\alpha_{1}\right)}}^{\left(\alpha_{1}\right)} A_{k^{\left(\alpha_{2}\right)}}^{\left(\alpha_{2}\right)}} \\
& \quad=\Gamma_{\alpha_{1} \alpha_{2}}^{\alpha_{2} \alpha_{1}} e^{i k^{\left(\alpha_{2}\right)}} A_{k}^{\left(\alpha_{2}\right)} A_{k^{\left(\alpha_{1}\right)}}^{\left(\alpha_{1}\right)},
\end{aligned}
$$

where $t_{\alpha_{1} \alpha_{2}}=1-\Gamma_{\alpha_{1}}^{0 \alpha_{1}}-\Gamma_{0}^{\alpha_{2} \alpha_{2}}-\Gamma_{\alpha_{2}}^{\alpha_{1} \alpha_{1}} \begin{array}{cc}\alpha_{2} & \text {. This two relations }\end{array}$ need to be identically satisfied since at this level we want to keep $k^{(\alpha)}$ as free complex parameters. Then Eq. (14) implies special choices of the coupling constants $\Gamma_{k l}^{m n}$,

$$
\Gamma_{2}^{1} \frac{2}{2}=\Gamma_{0}^{1} \frac{0}{0}, \quad \Gamma_{1}^{2} \frac{1}{2}=\Gamma_{1}^{0} \frac{1}{1} .
$$

The integrability conditions (15) reduce the two parameters defining the Hamiltonian (1) into only one free parameter $\Gamma_{0}^{1}{ }_{1}^{0} / \Gamma_{1}^{0} 10$. Moreover, in order for the model to be integrable, the particle moves over impurities as if the impurities are vacant sites. This condition will play a fundamental role in 
the spectral properties of the Hamiltonian (1), giving us the full spectrum of the standard ASEP plus new levels. From Eqs. (14) and (15) we obtain the structural constants,

$$
S_{2}^{2}{ }_{1}^{1}\left(k^{(2)}, k^{(1)}\right)=\frac{1}{S_{12}^{1} \frac{2}{\left(k^{(1)}, k^{(2)}\right)}}=e^{i k^{(2)}} .
$$

General $n$. We now consider the case of arbitrary numbers $n_{1}, n_{2}$ of particles of types 1 and 2 . The eigenvalue equation gives us generalizations of Eqs. (4) and (6). To solve these equations we identify the matrices $A^{(\alpha)}$ as composed of $n_{\alpha}$ spectral-dependent matrices (7). The configurations where $x_{i+1}>x_{i}+1$ give us the energy (8). The amplitudes in Eq. (2) where a pair of particles of types $\alpha_{1}$ and $\alpha_{2}$ are located at the closest positions give us the algebraic relations (9) where the algebraic structure constants are the diagonal $S$ matrix defined by Eqs. (10) and (16), and $S_{2}^{2}{ }_{2}^{2}\left(k_{j}^{(2)}, k_{l}^{(2)}\right)=1$, with coupling constants (15).

In order to complete our solutions we should fix the spectral parameters $\left(k_{1}^{(1)}, \ldots, k_{n_{1}}^{(1)}\right.$ and $\left.k_{1}^{(2)}, \ldots, k_{n_{2}}^{(2)}\right)$. Using the algebraic relations (9) for an arbitrary amplitude $A_{k_{1}^{(1)}}^{(1)} \cdots A_{k_{n_{1}}^{(1)}}^{(1)} A_{k_{1}^{(2)}}^{(2)} \cdots A_{k_{n_{2}}^{(2)}}^{(2)} E^{L}$ we obtain, from the periodic boundary condition,

$$
\begin{gathered}
e^{i k_{j}^{(1)} L}=-\exp \left(-i \sum_{l=1}^{n_{2}} k_{l}^{(2)}\right) \prod_{l=1}^{n_{1}} S_{1}^{1} \frac{1}{1}\left(k_{j}^{(1)}, k_{l}^{(1)}\right), \\
e^{i k_{q}^{(2)}\left(L-n_{1}\right)}=1,
\end{gathered}
$$

where we have $\left(j=1, \ldots, n_{1}\right)$ and $\left(q=1, \ldots, n_{2}\right)$. Finally, the momentum of the eigenstate is given by inserting the ansatz (7) into the relation $f\left(x_{1}, \alpha_{1} ; \ldots ; x_{n}, \alpha_{n}\right)=e^{-i P} f$ $\left(x_{1}+1, \alpha_{1} ; \ldots ; x_{n}+1, \alpha_{n}\right)$,

$$
P=\sum_{j=1}^{n_{1}} k_{j}^{(1)}+\sum_{j=1}^{n_{2}} k_{j}^{(2)}=\frac{2 \pi l}{L} \quad(l=0,1, \ldots, L-1) .
$$

Spectral gap. We can write the Bethe equation (17) in a more convenient way. From the second expression in Eqs. (17) we have

$$
\exp \left(-i \sum_{j=1}^{n_{2}} k_{j}^{(2)}\right)=e^{-i 2 \pi m /\left(L-n_{1}\right)}=\phi_{m},
$$

with $m=0,1, \ldots, L-n_{1}-1$. By inserting Eq. (19) and using Eq. (10) in the first equation in Eqs. (17) we obtain

$$
e^{i k_{j} L}=(-)^{n_{1}-1} \phi_{m} \prod_{l=1}^{n_{1}} \frac{\Gamma_{0}^{1}{ }_{1}^{0}+\Gamma_{1}^{0}{ }_{0}^{1} e^{i\left(k_{j}+k_{l}\right)}-e^{i k_{j}}}{\Gamma_{0}^{1}{ }_{1}^{0}+\Gamma_{1}^{0}{ }_{0}^{1} e^{i\left(k_{j}+k_{l}\right)}-e^{i k_{l}}},
$$

where $j=1, \ldots, n_{1}$ and $k_{j} \equiv k_{j}^{(1)}$. The Bethe equation (20) differs from the one related to the stochastic version of the asymmetric XXZ chain [8] by the phase factor $\phi_{m}$ defined in Eq. (19). However, different from the phase factor in the asymmetric XXZ with long-range interactions [11], the phase $\phi_{m}$ defined in Eq. (19) plays a fundamental role in the spectral properties of the model by changing the scaling behavior of the system. While in [11] the phase is a constant, in our model it is a function of the momentum of the state through relation (18). For $m=0$ we obtain the full spectrum of the standard ASEP [8]. Additional energy levels are given by different choices for $m$, and the first excited state belongs to these new levels.

Finally, it is important to notice that the Bethe equation (20) does not depend on the number of impurities $n_{2} \neq 0$. Consequently, the roots of Eq. (20) and the eigenvalues (8) are independent of $n_{2} \neq 0$ (but the wave function depends on $n_{2}$ ). This huge spectrum degeneracy follows from the conservation of the numbers of vacant sites between the impurities by the Hamiltonian (1). Let us explain with the following example. Suppose we start with the given configuration of 0120220 with one particle (1), three impurities (2), and three vacant sites $(0)$. We can make a surjective map between all possible configurations of these particles to all possible configurations of a new chain with just impurities and vacant sites. For example, $0120220 \Rightarrow 020220$. On this new chain, we are looking only for the effective movement of impurities on the chain. For simplicity, let us consider the totally asymmetric exclusion process (TASEP) model where $\Gamma_{0}^{1}{ }_{1}^{0}=1$ and $\Gamma_{10}^{0}{ }_{1}^{1}=0$. When the particle jumps over the impurities, nothing changes in the effective chain since we also have $0210220 \Rightarrow 020220$, then $0201220 \Rightarrow 020220$, then $0202120 \Rightarrow 020220$, then $0202210 \Rightarrow 020220$, then $0202201 \Rightarrow 020220$, and finally a change in the mapped configuration $1202200 \Rightarrow 202200$. In other words, the impurities move on the mapped chain as they are just one "object" due to the conservation of vacant sites between impurities. Moreover, this object only moves when the particle completes a turn over the chain. As a consequence, the time for the particle to complete one revolution is the time scale for the movements of this object. For an arbitrary number of particles in a chain of length $L$, the time for the particles to complete one revolution is of order $L^{3 / 2}$. As the object formed by the impurities needs to move of order $L$ times to span all possible configurations, we will need a time of order $L^{3 / 2} \times L=L^{5 / 2}$ to reach the stationary state. $[8]$

The Bethe equation (20) for the TASEP can be written as

$$
\left(1-z_{j}\right)^{n_{1}}\left(1+z_{j}\right)^{L-n_{1}}=-2^{L} \phi_{m} \prod_{l=1}^{n_{1}} \frac{z_{l}-1}{z_{l}+1} \equiv Y,
$$

where $z_{j}=2 e^{-i k_{j}}-1$, and we introduced an auxiliary complex variable $Y$. It can be noted that the right-hand side of these equations is independent of the index $j$; consequently, the Bethe equation (21) can be reduced to a simple polynomial equation of degree $L$ as in [8]. In the half-filling sector $n_{1}$ $=L / 2$ this polynomial is further simplified to $Y=\left(1-z^{2}\right)^{n_{1}}$. To solve this Bethe equation we follow the method developed in [8] and we pick a $Y$ and we take its $n_{1}$ th root. These roots give us $2 n_{1}$ solutions $z_{j}$. For each particular choice of $n_{1}$ out of $2 n_{1}$ solutions we have to determine $Y$ through Eq. (21). On the other hand, we also solve Eq. (21) numerically by direct computation of the roots of Eq. (21) up to $L=1024$. The stationary state is provided by choosing $m=0$ and $P=0$ and selecting the $n_{1}$ fugacities $z_{j}$ with the largest real parts. The first excited state is also obtained by selecting the $n_{1}$ fugacities with the largest real parts and by choosing $m=1$ 
and $P=\frac{2 \pi}{L}$. The energy gap has a leading behavior of KPZ $L^{-3 / 2}$ and a subleading term $L^{-5 / 2}$ related to the superdiffusion of particles 1 and subdiffusion of particles 2, respectively. For $m=0$ (and also for $n_{2}=0$ ) the subleading term vanishes and we recover the spectrum of the ASEP without impurities [8]. For $m=1$ the spectral gap scales with $L^{-5 / 2}$ instead of $L^{-3 / 2}$ due to the subleading term. It is important to notice that the dynamics of particles 1 is not affected by the impurities. This explains why the model displays the full spectrum of the ASEP. On the other hand, the dynamics of the impurities is totally dependent on the particles since the impurities only move when particles change positions with them. Consequently, the time to vanish the fluctuations on the densities of particle acts as a time scale for the diffusion of the impurities, resulting in a relaxation time longer than the one for the standard ASEP and reflected in the $L^{-3 / 2}$ $\times L^{-1}=L^{-5 / 2}$ gap.

Eigenstates. The MPA (3) enables us to write all eigenstates of Eq. (1) in a matrix product form. For a given solution $k_{j}$ and $k_{j}^{(2)}$, the matrices $E$ and $A_{k_{j}^{(\alpha)}}^{(\alpha)}$ have the following finite-dimensional representation:

$$
\begin{aligned}
& E=\otimes_{l=1}^{n_{1}}\left(\begin{array}{cc}
1 & 0 \\
0 & e^{i k_{l}}
\end{array}\right) \underset{l=1}{n_{2}}\left(\begin{array}{cc}
1 & 0 \\
0 & e^{i k_{l}^{(2)}}
\end{array}\right), \\
& \left.A_{k_{j}^{(2)}}^{(2)}={\underset{l=1}{n_{1}}(}_{l=1}\left(\begin{array}{cc}
1 & 0 \\
0 & e^{i k_{l}^{(2)}}
\end{array}\right)\right)_{l=1}^{j-1} \otimes I_{2} \otimes\left(\begin{array}{cc}
0 & 0 \\
1 & 0
\end{array}\right) \underset{l=j+1}{\otimes} I_{2},
\end{aligned}
$$

$$
A_{k_{j}}^{(1)}=\otimes_{l=1}^{j-1}\left(\begin{array}{ccc}
S_{1}^{1} & 1 & 1 \\
0 & \left(k_{j}, k_{l}\right) & 0 \\
0 & 1
\end{array}\right) \otimes\left(\begin{array}{ll}
0 & 0 \\
1 & 0
\end{array}\right) \underset{j=j+1}{\otimes_{1}+n_{2}} I_{2},
$$

where $n=n_{1}+n_{2}, I_{2}$ is the $2 \times 2$ identity matrix, and the dimension of the representation is $2^{n}$. The matrix product form of the eigenstates is given by inserting the matrices (7) defining the MPA (3) with the spectral parameters (20) into Eq. (2). As showed in [12], our MPA generalizes the steady-state matrix product introduced by Derrida et al. [13]. The stationary state is obtained by choosing $k_{j}=0$ and $k_{j}^{(2)}=0$ in Eq. (22). However, a relation between our matrix product form for the steady state and the standard matrix product form [13] is not trivial [12].

Finally, like in the ASEP, the stationary state is obtained by a combination of all possible configurations of particles and impurities with the same probability (satisfying the order $\left\{d_{1}, \ldots, d_{n_{2}}\right\}$ of vacant sites between impurities, up to cyclic permutations). This can be seen from the fact that the particles move on the chain as if the impurities are vacant sites and the impurities move on the surjective mapped chain as a single object. The spectral gap for other sectors and other boundary conditions and the physical consequences of these are currently under investigation.

We are grateful to G. M. Schütz and F. C. Alcaraz for the very helpful discussions.
[1] B. Sutherland, Phys. Rev. B 12, 3795 (1975).

[2] P. Schlottmann, Phys. Rev. B 36, 5177 (1987).

[3] F. C. Alcaraz, M. Droz, M. Henkel, and V. Rittenberg, Ann. Phys. 230, 250 (1994); G. M. Schütz, J. Phys. A 36, R339 (2003).

[4] V. Popkov, M. E. Fouladvand, and G. M. Schütz, J. Phys. A 35, 7187 (2002).

[5] M. J. Lazo, Braz. J. Phys. 38, 237 (2008).

[6] F. C. Alcaraz, D. Arnaudon, V. Rittenberg, and M. Scheunert, Int. J. Mod. Phys. A 9, 3473 (1994); D. Arnaudon and Z. Maassarani, J. High Energy Phys. 1998, 024 (1998).

[7] F. C. Alcaraz and M. J. Lazo, J. Phys. A 39, 11335 (2006); 37, L1 (2004); 37, 4149 (2004); Braz. J. Phys. 33, 533 (2003).
[8] L. H. Gwa and H. Spohn, Phys. Rev. Lett. 68, 725 (1992); Phys. Rev. A 46, 844 (1992).

[9] M. Kardar, G. Parisi, and Y. C. Zhang, Phys. Rev. Lett. 56, 889 (1986).

[10] It is important to mention that as we have two distinct sets of spectral parameters, different from the models in [6], our model will not have a $R$ matrix on the difference of spectral parameters.

[11] F. C. Alcaraz and R. Z. Bariev, Phys. Lett. A 240, 247 (1998); Phys. Rev. E 60, 79 (1999).

[12] O. Golinelli and K. Mallick, J. Phys. A 39, 10647 (2006).

[13] B. Derrida, M. R. Evans, V. Hakim, and V. Pasquier, J. Phys. A 26, 1493 (1993). 